\title{
UNA NUEVA CONCEPCION DE LA MATERIA \\ EL MODELO ESTANDAR
}

\author{
Julián Urrea B. \\ Profesor Asociado \\ Departamento de Física \\ Universidad Pedagógica Nacional
}

Una imagen más comprensible de la naturaleza y una visión unificada de la física pueden ser presentadas a los estudiantes del bachillerato, gracias al conocimiento de las componentes fundamentales de la materia y de sus interacciones, desarrollado en los últimos 25 años.

\section{Introducción*}

El Modelo Estándar es un modelo de la naturaleza, de las componentes elementales de la misma y de sus fuerzas de interacción.

Hasta hoy no ha habido experimentos cuyos resultados estén en desacuerdo con el Modelo Estándar oque no puedan ser explicados por él.

Sin necesidad de usar el formalismo matemático propio de la Física de las partículas elementales, se puede describir el Modelo Estándar a estudiantes de educación secundaria. Sabemos muy bien que la presentación de algunos hechos relacionados con los avances de la Física puede hacerse con menos dificultades que las observadas en la enseñanza de la Física siguiendo la evolución de los conceptos.

Más aún, pedagógicamente es conveniente explicar las ideas fundamentales del Modelo Estándar sin la pretensión de seguir un proceso histórico, pues no es posible atribuir este modelo a ningún físico en particular ni tampoco asignar una fecha específica a su primera formulación teórica(1). Solamente se sabe que a partir de 1975 distintas componentes del Modelo Estándar empezaron a conformar la estructura final que hoy se conoce.

No se abordará tampoco la problemática que dio origen al Modelo Estándar, pero es posible aludir a la situación existente hasta 1974 con las palabras de Steven Wiemherg, uno de los más eminentes físicos que contribuyeron a su creación: "No hay ahora ninguna teoría satisfactoria que explique las partículas elementales en términos de componentes más simples."(2).

Por lo demás, es un hecho que la Física de Partículas indaga sobre objetos sin historia, y trata de explicar conceptos creados por la inteligencia del hombre que ineludiblemente se refieren a un universo físico muy tangible(3).

\section{Partículas Elementales}

\footnotetext{
* Trabajo presentado en el Sexto Coloquio Distrital de Física. Julio de 1991
} 
Entre los años de 1869 y 1871 Dimitri Mendeleyev formuló un ordenamiento de los elementos en la tabla periódica. En ella aparecen regularidades, inexplicables en aquella época, que hicieron posible la predicción de la existencia de elementos desconocidos, mucho antes de que la Mecánica Cuántica diera una explicación satisfactoria de la tabla periódica.

La versión moderna de un ordenamiento de partículas elementales es el Modelo Estándar, en que el ordenamiento se refiere a tres clases de partículas: los quarks, los leptones y los bosones de intercambio, es decir, a dos clases de partículas indivisibles (Tabla 1) y a una clase de portadores de las fuerzas de interacción.

\section{Tabla 1}

\section{Partículas Elementales}

Símbolos y nombres de las componentes fundamentales de la materia

\begin{tabular}{|l|c|c|c|}
\hline \multirow{4}{*}{ Quarks } & $\mathrm{u}$ & $\begin{array}{c}\mathrm{c} \\
\text { Charm }\end{array}$ & $\begin{array}{c}\mathrm{t} \\
\text { truth }\end{array}$ \\
\cline { 2 - 4 } & $\mathrm{Up}$ & $\mathrm{s}$ & $\mathrm{b}$ \\
$\mathrm{d}$ & strange & bottom \\
\hline \multirow{3}{*}{ Leptones } & $\mathrm{e}$ & $\mu$ & $\tau$ \\
& electrón & Muón & tau \\
\cline { 2 - 4 } & $\mathrm{V}_{\mathrm{e}}$ & $\mathrm{v}_{\mu}$ & $\begin{array}{c}\mathrm{v}_{\tau} \\
\text { tautónico }\end{array}$ \\
\hline
\end{tabular}

Entre las 12 componentes elementales de la naturaleza figura el electrón, descubierto en 1900, el muón, descubierto en 1925 y los neutrinos, dos de ellos descubiertos en 1962.

Aunque se desconocen aún las masas de los neutrinos, se asume que las 12 partículas tienen masa, en tanto que sólo 9 de ellas tienen carga; los neutrinos tienen carga cero. Si incluimos estas propiedades de las partículas en la tabla 1 se obtiene la tabla 2.

\section{Tabla 2}

Masa y Carga de las Partículas Elementales

La masa que aparece bajo el símbolo de cada partícula está dada en MeV. El último número de cada casilla corresponde a la carga de la partícula tomando como unidad la carga del electrón.

\begin{tabular}{|c|c|c|}
\hline $\mathrm{u}$ & $\mathrm{c}$ & $\mathrm{t}$ \\
300 & 1500 & $\mathrm{i}$ \\
$2 / 3$ & $2 / 3$ & $2 / 3$ \\
\hline $\mathrm{d}$ & $\mathrm{s}$ & $\mathrm{b}$ \\
\hline
\end{tabular}




\begin{tabular}{|c|c|c|}
\hline 300 & 500 & 4500 \\
$-1 / 3$ & $-1 / 3$ & $-1 / 3$ \\
\hline $\mathrm{e}$ & $\mu$ & $\tau$ \\
0.5 & 106.7 & 1780 \\
-1 & -1 & -1 \\
\hline $\mathrm{V}_{\mathrm{e}}$ & $\mathrm{V}_{\mu}$ & $\mathrm{V}_{\tau}$ \\
$<3 \times 10^{-5}$ & $<15 \times 10^{-2}$ & $<90$ \\
0 & 0 & 0 \\
\hline
\end{tabular}

Cada columna de la tabla 2 se llama GENERACION. La masa de cada partícula o su límite experimental se da en electron-voltios en tanto que su carga se da tomando como unidad la carga del electrón. La carga mínima corresponde a los quarks d,s y b.

Solamente la primera generación está formada por partículas estables, es decir que el universo está constituido por quarks u, quarks d y por electrones (Fig.1).

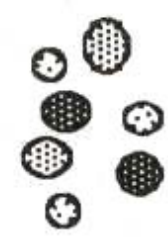

Quarks

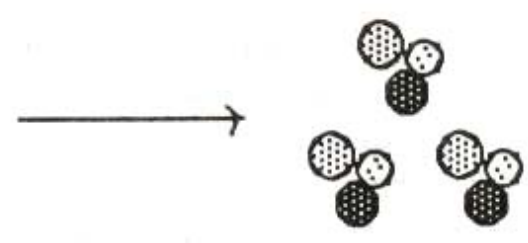

Protones y Neutrones

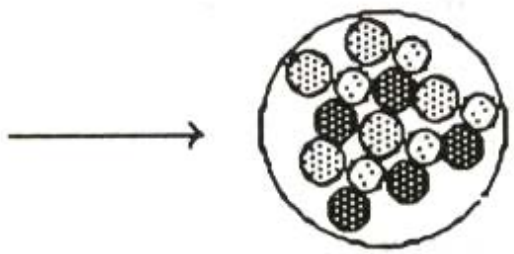

Núcleo

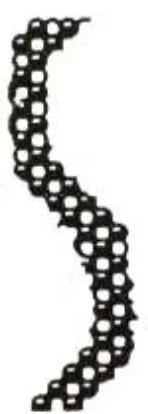

Macromolécula

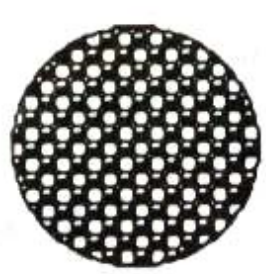

Molécula
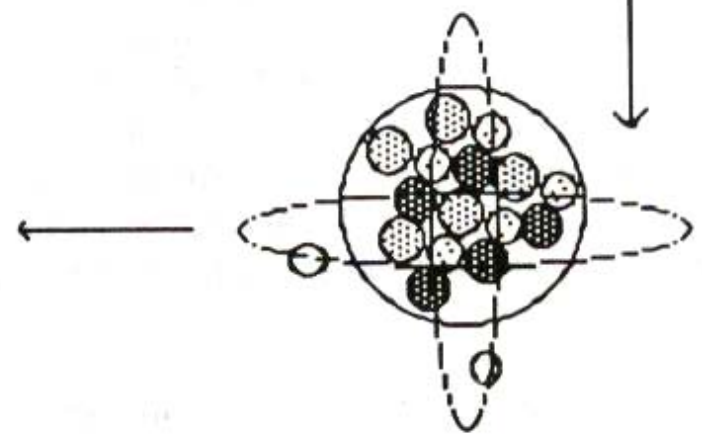

Atomo

Figura 1. Configuraciones de la materia desde los quarks hasta las grandes moléculas.

Aunque no aparece en la tabla No. 2, las 12 partículas tienen spin 1/2, es decir momento angular intrínseco semi-entero que se mide en unidades de $h / 2 \pi$, donde $h$ es la constante de Plank (1.05 $\times 10^{-34}$ Julios.s).

En razón de este momento angular intrínseco las partículas que tienen spin semientero se llaman fermiones; el electrón es un fermión, por ejemplo. Las partículas que tienen spin entero se llaman bosones; tal es el caso del fotón.

\section{Constantes de Interacción}


Las fuerzas o interacciones fundamentales en la naturaleza son: la interacción fuerte, la interacción electromagnética, la interacción débil y la interacción gravitacional. La primera es responsable de la estabilidad del núcleo manteniendo en su interior los nucleones (protones y neutrones); la segunda lo es de la estabilidad del átomo haciendo que los electrones cargados negativamente se hallen en cierta proximidad del núcleo; la fuerza débil aparece en la llamada desintegración $\beta$ (emisión nuclear de un electrón y un anti-neutrino) y finalmente la interacción gravitacional que se observa en la atracción de masas (4).

Los quarks pueden interactuar a través de los cuatro tipos de fuerzas y los leptones cargados, electrón, muón y tau, solamente con tres de las fuerzas mencionadas: la fuerza electromagnética, la fuerza débil y la fuerza gravitacional.

Las interacciones entre partículas son el único medio de que disponemos para saber de su existencia y de sus propiedades. Por esta razón los conceptos de partícula y de interacción son inseparables.

Una constante de interacción es una cantidad que indica la intensidad de la fuerza; por ejemplo, con relación a las interacciones gravitacional y electromagnética, las constantes $\mathrm{G}$ y $\mathrm{K}$ son las que aparecen en la expresión matemática de la ley de la gravitación universal y de la ley de Coulomb:

$$
\begin{aligned}
& F G=G \quad \frac{m_{1} m_{2}}{R^{2}} \\
& F E=K \quad \frac{q_{1} q_{2}}{R^{2}}
\end{aligned}
$$

La intensidad relativa de las fuerzas puede compararse utilizando únicamente las constantes de interacción. Por ejemplo si se mide la intensidad de las cuatro interacciones entre dos quarks, fuerte, electromagnética, débil y gravitacional, la relación entre ellas es, en este orden, como

$$
1.0: 0.01: 10^{-9}: 10^{-43}
$$

\section{Cargas y Fuerzas}

El concepto de carga, la idea familiar del electromagnetismo, puede generalizarse a las demás interacciones fundamentales si redefinimos "carga" como fuente de fuerza. En este sentido amplio, la interacción gravitacional se origina en una sola "carga", es decir, en la masa; y la fuerza electromagnética en dos cargas, positiva y negativa.

En la interacción fuerte se presentan tres clases de carga que, por cierta analogía con los colores, reciben el nombre de rojo, verde y azul (Fig.2); y se ha establecido que los quarks se atraen siempre que la carga de color resultante sea blanco. Las únicas combinaciones posibles de los quarks serán aquellas en que la estructura resultante sea neutra ya que un exceso de carga de color rojo, verde o azul no existe. Es imposible 
separar un quark de una combinación estable, según lo afirma un principio llamado de confinamiento.

Una primera combinación posible se llama bailón y está formada por tres quarks qqq:

$$
\text { rojo }+ \text { verde }+ \text { azul }=\text { blanco }
$$

La segunda estructura posible se llama mesón y resulta de la combinación de un quark y un antiquark qq:

$$
\text { rojo }+ \text { anti }- \text { rojo }=\text { blanco }
$$

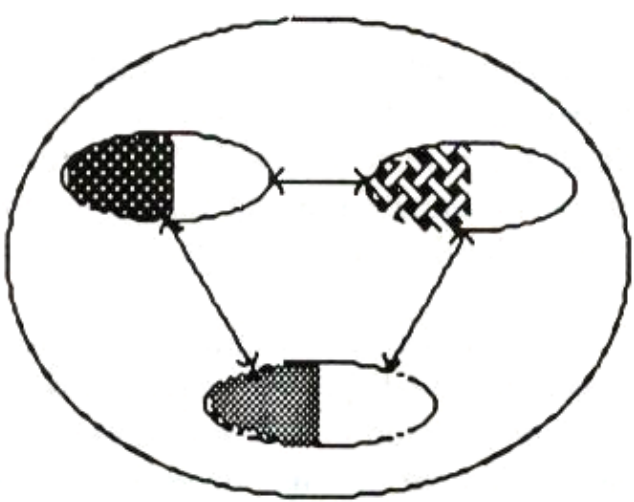

Protón

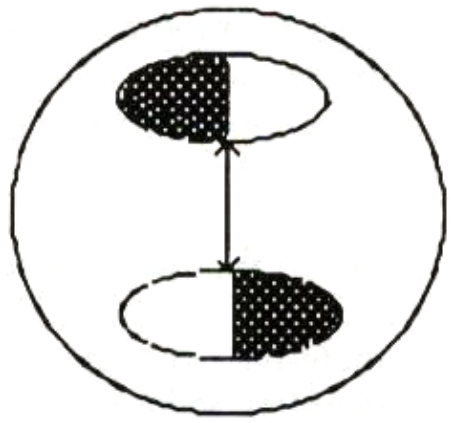

Mesón

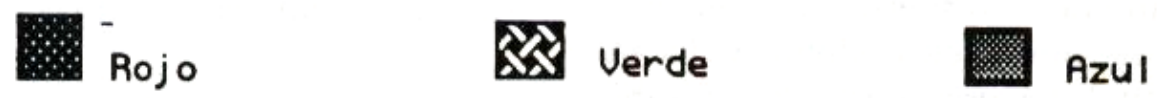

Figura 2. Los protones y los neutrones se componen de tres quarks de diferentes colores, en tanto que los mesones se componen de un quark de cierto color y de un antiquark del anticolor correspondiente.

\section{Los Bosones de Intercambio}

Hasta ahora solamente hemos mencionado quarks y leptones como componentes fundamentales de la materia. Pero es un hecho establecido firmemente en el laboratorio que las interacciones fundamentales ocurren por intercambio de ciertas partículas llamadas, por esta razón, bosones de intercambio. Por ejemplo el bosón de la interacción electromagnética es el fotón.

Un quark interactúa con un portador de fuerza de color mediante la partícula de intercambio llamada gluón (Fig.3). Esta partícula es la portadora de la carga de color, situación diferente de la que se observa en el fotón que no es portador de ninguna carga. 


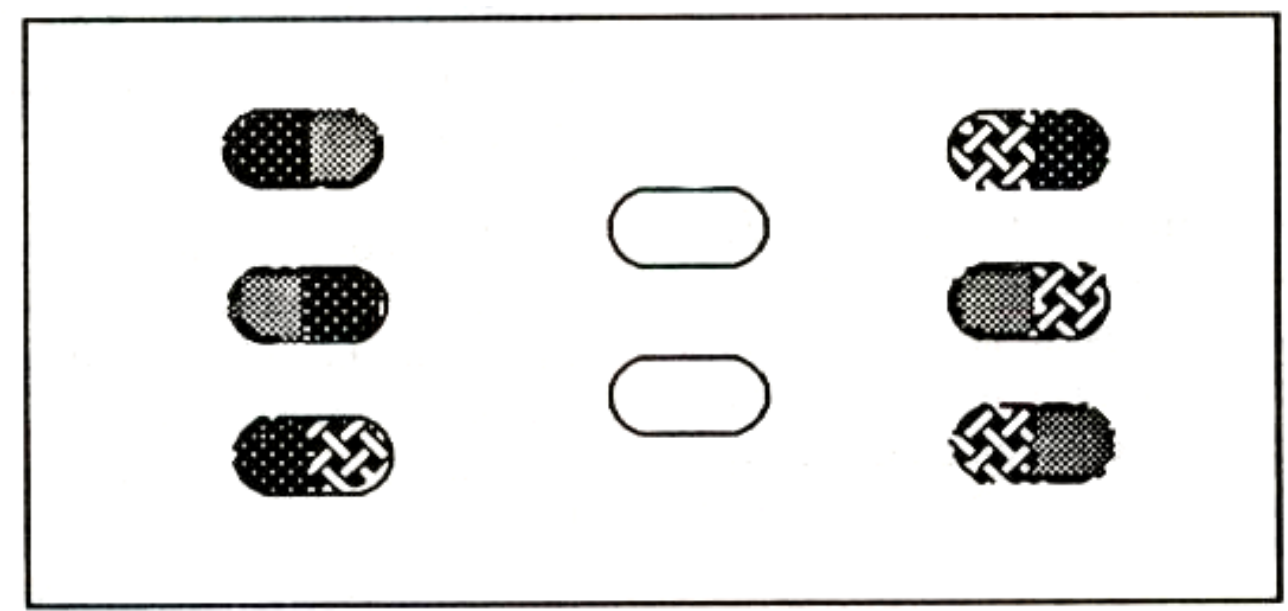

\section{Gluones}

\section{Rojo Barde Verde Azul}

Figura 3. Cada gluón es portador de un color y de un anticolor. Los gluones son responsables de la interacción de un quark con otro portador cualquiera de fuerza de color. En esta figura, como en la anterior, un anticolor se representa por el color correspondiente en la posición opuesta.

La fuerza entre dos quarks aumenta a medida que aumenta la distancia que los separa. Este hecho curioso es "crucial" en el sentido de que la hipótesis de la carga de color explica claramente la razón por la cual no es posible aislar un quark. Para aislar un quark de la configuración estable a la que está unido sería necesaria una energía infinita.

\section{La Unificación Electrodébil}

Hacia la mitad de la década de los ochentas se logró la comprobación experimental de una teoría, también reciente, conocida como la teoría de la unificación electrodébil, esquema de unificación de la fuerza electromagnética y de la fuerza nuclear débil.

Según la teoría, estas dos fuerzas son una misma interacción, aunque ofrecen una fenomenología diferente a causa de la existencia de masa en los portadores de la interacción débil y de la ausencia de masa en el fotón, que es el portador de la interacción electromagnética. Un portador de la interacción débil tiene una masa equivalente a la masa del protón por un factor de cien (100).

De este modo resulta fácil comprender porqué el fotón puede viajar cualquier distancia a la velocidad de la luz sin degradarse, en tanto que el bosón de la interacción débil se degrada antes de salir del núcleo atómico. Esto explica, además, el porqué del corto 
alcance de la interacción débil y el largo alcance de la interacción electromagnética, cuya intensidad disminuye con el cuadrado de la distancia.

El carácter neutro del fotón sugirió a los creadores de la teoría electrodébil la existencia de corrientes de bosones neutros Zo y de corrientes de bosones cargados $\mathrm{W}^{+}$y $\mathrm{W}^{-}$, llamados en conjunto bosones vectoriales intermedios.

La elegancia formal de la teoría y el ingenio sutil de los experimentos realizados para su comprobación fueron suficientes para que sus autores Steven Wiemberg, Abdus Salam y Sheldon Glashow recibieran el premio Nobel de Física en el año de 1975.

Así mismo un equipo de 130 físicos experimentales del CERN, dirigidos por Carlo Rubbia y Simon Van der Meer, observaron los bosones vectoriales intermedios y midieron sus masas que, para sorpresa de todos, resultaron exactamente iguales a las masas esperadas según la teoría de Wiemberg-Salam. También Rubbia y Van der Mier fueron galardonados con el premió Nobel de Física en el año de 1983.

\section{Conclusión}

En síntesis, podemos concluir que a la luz de la física contemporánea, protones, neutrones y mesones no son componentes indivisibles de la materia como se pensó en un comienzo. Las componentes fundamentales son doce fermiones subdivididos en dos grupos formados por seis quarks y seis leptones respectivamente.

Los quarks interactúan entre sí gracias a que tienen tres tipos de carga llamados cargas de color rojo, azul y verde por la analogía que existe entre la ausencia de color al combinar estos tres colores y la ausencia de carga de color en los compuestos estables o bariones.

Los quarks que forman tales estructuras solamente se combinan en la forma qqq con resultado de carga de color neta igual a cero en las partículas conocidas hasta hoy. La ausencia de carga de color de los bationes es análoga a la carencia de color que se obtiene de la combinación rojo, azul y verde (5).

En las interacciones electromagnética, débil y fuerte intervienen doce bosones o portadores de fuerzas; estos son: el fotón, los bosones vectoriales intermedios $\mathrm{W}^{+}, \mathrm{W}^{-}, \mathrm{y}$ $Z_{o}$ y ocho gluones. No se ha tenido en cuenta la interacción gravitacional ni su portador, el gravitón.

Las consideraciones anteriores solamente han tenido en cuenta el microcosmos o espacio interior. Para satisfacción nuestra, la identificación de las partículas elementales es en nuestros días un problema que interesa por igual a la Física de Partículas y a la Cosmología, la ciencia por excelencia del Universo o espacio exterior, porque el desarrollo experimental de los 5 últimos decenios, sólo comparable con la evolución vertiginosa de la teoría, avanza con igual intensidad hacia el descubrimiento de lo muy grande y de lo muy pequeño, del origen del Universo y de la constitución última de la materia.

Todos los esfuerzos que realicemos para comprender la relación entre el experimento y la teoría nos acercan un poco más a una nueva concepción del Universo porque esa relación es "el corazón mismo de lo que se llama método científico" (6). 


\section{Referencias}

1. Jovanovic, Drasko, The Standard Model, Education Programs at Fermilab, Batavia, Illinois, 1990.

2. Weimberg, Steven, citado por D. Jovanovic, op. cit. pag. 4.

3. Laberrigue, J., Paty, M., Les physiciens des particules en quête de leur histoire, La Recherche No135, pag. 908, 1982.

4. Mujin, K., Física Nuclear Recreativa, Editorial Mir, Moscú, 1985.

5. Feynman, R. P., Física, Vol. I, Ed. Fondo Educ. Interamericano, Bogotá, 1971.

6. Lederman, L., From, .Quarks to the Cosmos, Scientific American Library, New York, 1989. 